\title{
GESCHICHTE ALS KOMMUNIKATIONSPROBLEM ${ }^{*}$
}

Die Erforschung der Geschichte der Kommunikation ist selbst ein historisch junges Phänomen. Bis ins späte I9. Jahrhundert wurde Kommunikation nicht als eigenständiger, vom Transport, der Öffentlichkeit, der Zirkulation, der Sprache oder dem Sprechen abgekoppelter Bereich verstanden. Die Vorstellung, dass es etwas wie Kommunikation gäbe und dass sie eine Geschichte haben könnte, tauchte in der Geschichts- und Wirtschaftswissenschaft des I9. Jahrhunderts mit Figuren wie Tocqueville und Guizot in Frankreich, Mill in England sowie Knies und Schäffle in Deutschland auf. Zwei repräsentative Figuren, die diese Arbeit im 20. Jahrhundert soziologisch gefestigt haben, waren Charles Horton Cooley in den Vereinigten Staaten und Werner Sombart in Deutschland. Cooleys Diktum, dass «Transport physisch, Kommunikation psychisch» sei, markiert einen Umschlagpunkt des langsamen Übergangs des Konzeptes der Kommunikation von materiellen zu metaphysischen Modi der Beförderung. ${ }^{1}$ Um I930 gewann die Mediengeschichte mit Denkern wie Lewis Mumford, John Dewey, Edward Sapir, Walter Benjamin und dessen Mitstreitern der Frankfurter Schule an Gestalt. Aber die Schlüsselfigur bildet wohl weiterhin der kanadische Wirtschaftshistoriker Harold Adam Innis (1 894-1952), der der Mediengeschichte in seinen letzten, krebsgezeichneten Lebensjahren eine Serie von Essays, Büchern und ein unvollendetes Konvolut von Manuskripten gewidmet hat. Innis' Karriere begann mit der Untersuchung des Umlaufs von Gütern und endete mit der Erforschung von Medien, wobei die Rolle von Netzwerken und die Materialitäten des Austausches seine Arbeit dauerhaft prägten. Damit trug er dazu bei, die symbolischen Aspekte des Konzepts der Kommunikation hervortreten zu lassen.

Viele seiner Kollegen scheinen es anfangs für eine verschrobene Idee Innis' gehalten zu haben, die Medien der Kommunikation auf die gleiche Stufe zu stellen wie die traditionellen Antriebskräfte der Weltgeschichte: Politik, Ökonomie,
* Gekürzte Fassung des Aufsatzes: History as a Communication Problem, in: Barbie Zelizer (Hg.), Explorations in Communication and History, London (Sage) 2008, 19-34. 1 Eine Übersicht über diese Geschichte bietet mein Artikel: Communication, History of the Idea, in: Wolfgang Donsbach (Hg.), International Encyclopedia of Communication, Oxford (Blackwell) 2008. 
2 Harold Adams Innis, The Bias of Communication, in: ders., The Bias of Communication, Toronto (University of Toronto Press) 1949, 33.

3 Anm. des Übersetzers: Zur Schwierigkeit der Übersetzung des Begriffs bias vgl. Karlheinz Barck, Harold Adam Innis, in: ders., Harold A. Innis, Wien (Springer) 1997, 3-13: «Man könnte bias im Deutschen vielleicht mit ‘Dreh', «Schere) oder 〈Kniff` übersetzen. Innis beschreibt damit die durch die Doppelgestalt aller Kommunikationsmedien, die sowohl Realität konstituieren als auch selbst Realität sind, vorgegebene Wahl zwischen unterschiedlichen Richtungen. Da Medien sich nicht gegenseitig auslöschen, vervielfältigt und kompliziert sich die Wahl zwischen den Möglichkeiten und ihrer Verwendung", 13 .
Krieg, Demografie und Kultur. In der Tat wirken Innis Ausführungen über seinen Zugang zum Aufstieg und Fall von Zivilisationen gelegentlich etwas monomanisch. Kleinlich gelesen, erscheint Innis Erkenntnis, dass die orale Tradition, Steintafeln, Papyrus, Pergament und Papier je unterschiedliche soziale und politische Welten und Arten der Aufzeichnung produzieren, durchaus banal. Liest man ihn hingegen großzügig, wird deutlich, dass Innis dem Repertoire des Historikers nicht einfach ein weiteres Motiv hinzugefügt hat. Denn als die Kommunikation einmal in die Geschichtswissenschaft eingeführt war, bedrohte sie - oder versprach sie -, deren Unternehmen zu revolutionieren. I949 schrieb er: «Our knowledge of other civilizations depends in large part on the character of the media used by each civilization insofar as it is capable of being preserved or of being made accessible by discovery.» ${ }^{2}$ Unser Wissen von der Vergangenheit ist eine Frage der Medien. Innis hat nicht nur die Mediengeschichte erfunden, sondern auch die Medien der Geschichte entdeckt.

Für Innis ist Geschichte ein Problem der Kommunikation über Raum und Zeit, und jedes Medium hat seine eigene Selektivität der Übertragung, Speicherung und Verfügbarmachung. Jedes Medium der Geschichte - Dokumente, Ruinen, Haushaltsgegenstände, Knochen, DNA oder was auch immer die Reise von der Vergangenheit in die Zukunft überstanden hat - leistet einen eigenständigen bias. ${ }^{3}$ Historiker, die lange Zeiträume untersuchen, legen ihren Fokus, so Innis, meist übermäßig auf die Religion. Sie vernachlässigen allzu schnell die Bürokratie, weil die Dokumente und Quellen, die ihnen zur Verfügung stehen, von auf Dauerhaftigkeit und Beständigkeit bedachten Agenten angelegt sind, etwa Priestern oder Geistlichen, und nicht von raumorientierten Agenten wie Richtern oder Händlern. Die Wahl des Themas und der Methode spannt das Dokument ein. Die Vergangenheit zu interpretieren heißt nicht nur die Inhalte historischer Quellen zu studieren, sondern ebenso die Konstitution dieser Quellen selbst. Bias impliziert nicht nur potentielle Gefahren für die Objektivität: Innis hat auch die textile Metapher eines schrägen Zuschnitts in Betracht gezogen. Historiker lesen gezwungenermaßen entlang der Diagonalen. Insofern sie die Bedingungen ihrer eigenen Praxis reflektieren, sind sie notwendig Medienwissenschaftler.

Medienwissenschaftler haben schon lange die Bedeutung der Historiografie sondiert. Beide Felder, die Medienwissenschaft wie die Geschichte, sind mit dem methodischen Problem konfrontiert, wie sich unter Bedingungen der Entfernung und Entfremdung überhaupt interpretieren lässt. Sie teilen ein auffallend ähnliches Vokabular der Quellen, Dokumente und Überlieferungen. Auch wenn die Medienwissenschaft sich typischerweise auf die Verteilung im Raum konzentriert hat, erlauben Medien ebenso die Ausbreitung über die Zeit. Übertragung und Speicherung, die Überwindung von Zeit und Raum sind zentrale Themen beider Felder. Speicherung ist ein Vorgang der Einschreibung von etwas in dauerhafter Form; Übertragung ist der Vorgang des Sendens von etwas Gespeichertem über eine Distanz, ob Zeit oder Raum; und Interpretation ist der Vollzug des 
Empfangens einer übertragenen Speicherung sowie ihre Verarbeitung in der Gegenwart. Historische Forschung ist immer mit der Triangulation von Speichern, Übertragen und Interpretieren beschäftigt. In diesem Essay schlage ich vor, die Konvergenz der Geschichtsphilosophie und der Medientheorie in der Hoffnung zu untersuchen, dass sich nicht nur das Verständnis der Medien- und Kommunikationsgeschichte erweitern lässt, sondern auch zu betonen, wie zentral die Probleme der Mediengeschichte für die Geschichtswissenschaft sind. Medien- und Kommunikationsgeschichte sind nicht bloß Supplement oder Hilfswissenschaft der Geschichtsforschung; sie sind eine Herausforderung unseres Umgangs mit Geschichte selbst.

Historiker haben bereits eine besondere Empfänglichkeit für Fragen der Medialität. Ihr Geschäft besteht darin, Dokumente in Bezug auf ihre Zeit, ihre Herkunft, ihren Autor, ihre Authentizität, ihre Tradition und so weiter auszuwerten. Die erste Frage eines Historikers an eine Quelle lautet nicht Was sagt sie? sondern Wie ist sie entstanden? oder sogar darüber hinaus Wie sind sie bierbin gekommen? Die Tatsache, dass die Quelle überhaupt existiert, ist vielleicht die aufschlussreichste Tatsache. Alle Historiker sind Medienwissenschaftler in dem Sinne, dass sie Texte und Artefakte hinsichtlich ihrer Prozesse der Produktion und Distribution lesen. Die Vergangenheit ist um den Riss zwischen Vergangenheit und Gegenwart angeordnet und wird von eben jenen historischen Prozessen geformt, die wir zu verstehen versuchen. Historiker, in anderen Worten, sind hochsensibel für die Bedingungen des Codierens und Decodierens. Der Kulturhistoriker Carlo Ginzburg spricht von einer Semiotik der Spuren und der Philosoph Paul Ricœur von einer Hermeneutik der Zeugenschaft. ${ }^{4}$ Beide Weisen der Interpretation konzentrieren sich auf Nebensächlichkeiten und Kontingenzen. Man kann die Nebenstatt der Hauptdarsteller betonen oder nicht auf die Sprache, sondern auf die Versprecher hören. Ginzburg und Ricœur verweisen auf einen forensischen Gestus, der Indizienbeweise und Symptome schätzt. Ein Historiker wird sich bei einem ersten Blick auf ein Dokument nicht gleich den Inhalten zuwenden; er oder sie wird sich der Herkunft, dem Bündel, der Ordnung der Bestandteile, der Falten annehmen. Historiker, die sich mit dem Druck beschäftigen, werden die Bindung eines Buches interessanter finden als seinen Text, ebenso wie Medizinhistoriker, die sich mit der Ausbreitung der Cholera in Europa beschäftigen, vielleicht eher ihre Nase in die Dokumente stecken werden, um herauszufinden, ob sie nach Essig riechen (der als Desinfektionsmittel gegen die Krankheit benutzt wurde), statt sie im herkömmlichen Sinn zu lesen. ${ }^{5}$ Das Medium ist auch in der Geschichtswissenschaft die Botschaft.

\section{Die historische Aufzeichnung}

Es ist das Ungewöhnliche, das dokumentiert wird. Das ist der bias, den Journalisten lieben: Mann beißt Hund. Aufzeichnungen sind grundsätzlich fehlbar und un-
4 Carlo Ginzburg, Clues: Roots of an Evidential Paradigm, in: ders., Myths, Emblems, Clues, London (Hutchinson Radius) 1990, 96-125 sowie Paul Ricœur, The Hermeneutics of Testimony. Essays in Biblical Interpretation, London (SPCK) 1981.

5 Beide Beispiele stammen aus Anthony Grafton, Dreams of a Universal Library, in: The New Yorker, 5. Nov. 2007, 50-54. 
vollständige Bewahrungen von Ereignissen. Selbst in der Alltagssprache, die als eine Art orale Dokumentation von Handlungen und Gedanken verstanden werden kann, ist das Verhältnis zwischen dem, was unterstellt und verstanden, und dem, was wirklich gesagt wird, riesig. Nur ein verschwindender Teil des gemeinsamen Verständnisses wird überhaupt im Sprechen artikuliert. Alle Dokumente stehen vor einem nicht wahrgenommenen Hintergrund.

Harold Garfinkel hat diese Einsicht auf institutionelle Praktiken der Speicherung angewandt. Dokumente, beispielsweise medizinische Akten, werden nicht unter der Perspektive einer vollständigen und umfassenden Wiedergabe für einen Historiker angelegt. Was für einen Beobachter unvollständig erscheinen mag, kann in institutionellen Routinen glänzend funktionieren. Es gibt, wie Garfinkel schreibt, «<gute〉 organisatorische Gründe für «schlechte〉 klinische Akten». ${ }^{6}$ Psychiatrische Berichte etwa zeigen nicht die alltägliche soziale Interaktion in einer Klinik; sie setzen sie vielmehr voraus und verbergen sie dadurch. Denkt man sie als getreue Spiegel, mögen sie verzerrend erscheinen, aber für den internen Gebrauch funktionieren sie tadellos. Die wichtigsten Informationen werden oft gerade nicht aufgezeichnet. Hierbei handelt es sich um eine Art kognitiver Ökonomie, um eine Politik und Soziologie der Dokumentation. Selten oder vielleicht nie wurde eine historische Aufzeichnung für Historiker angefertigt, die sich normalerweise (wie die meisten von uns in der Kommunikation) in der Position des Lauschers befinden. Diese Konstellation ist aus der Hermeneutik geläufig: wir lesen Texte, die nie an uns adressiert wurden. ${ }^{7}$

Texte ändern sich mit ihrem Transkriptionsprotokoll. Einmal kam ein Kollege in mein Büro und pries ein Sonderangebot an, das mit der Post gekommen war. Mit einem zynischen Grinsen auf dem Gesicht kündigte er ein neues Video der <Größten Momente in der Geschichte des Sports〉 an. Ich brauchte nur einen Moment, um das zu durchschauen: Wenn es ein Video ist, impliziert das den Ausschluss allen Sports vor der Erfindung der Filmkamera und außerhalb der Sichtweite einer solchen. Die Geschichte des Sports war plötzlich auf die Periode von I 895 bis heute geschrumpft. Aber ich machte einen subtileren Fehler: Ich hatte mich auf die Vorstellung eingelassen, dass es eine kontinuierliche Sportgeschichte gäbe, die von den Medien unberührt wäre, mit denen sie aufgezeichnet wird. Die Wahl des Mediums bestimmt die historische Aufzeichnung im grundlegendsten Sinn.

Ein weiterer raffinierter Fall sind archäologische Funde aus prähistorischer Zeit. Die Speicherung ist durch Äxte, Dolche, Pfeilspitzen und andere dauerhafte Din-

6 Harold Garfinkel, Studies in Ethnomethodology, Englewood Cliffs, New York (Prentice-Hall) 1967, bes. Kap. 2, 3 und 6 .

7 Paul Ricœur, Hermeneutics and the Human Sciences, Cambridge (Cambridge University Press) 1981. ge befangen. Solche Dinge sind mit Lewis Mumford eher power technologies als container technologies wie Mülleimer, Speicherkammern, Sprache, Familien, Reservoirs oder Rituale, die nur wenige Spuren hinterlassen. Werkzeuge überstehen die Zeit besser als Worte, Taten, Gedanken oder Praktiken der Kindererziehung. Dieses bruchstückhafte Überleben gibt uns, wie Mumford anmerkt, eine verzerr- 
te, ziemlich maskuline Sicht auf Menschen als werkzeugbenutzende Tiere anstatt etwa träumende oder sprechende Tiere. ${ }^{8}$ Was die Zeit mit den Aufzeichnungen gemacht hat, muss zugleich mit ihnen gelesen werden und könnte von einengenden Ideologien befreien.

Aufzeichnungen sind Beschreibungen, und es liegt in der Natur von Beschreibungen, unausschöpflich zu sein. Die mögliche Kommunikation über ein Ereignis ist niemals komplett. Es gibt immer noch etwas zu sagen, und eine Aufzeichnung ist per definitionem niemals abgeschlossen. Jeder Sprecher einer Sprache hat die beeindruckende Fähigkeit, verständliche Sätze von sich zu geben, die nie zuvor in der Geschichte gesagt wurden, und jeder Sprecher vollführt solche Kunststücke mehrmals täglich. Diese Generierbarkeit gilt ebenso für Dokumentationen wie auch für Beschreibungen. Keine Aufzeichnung ist jemals das letzte Wort. Das heißt natürlich nicht, dass manche Aufzeichnungen nicht detailreicher wären als andere. Wir können Samuel Pepys und Margaret Ballard dankbar sein, dass sie Einblicke in ihre Welt gegeben haben, aber niemand würde behaupten, dass ihre Tagebücher alles über London im I 7. oder Maine im I 8. Jahrhunderts verraten. ${ }^{9}$ Wir wissen nie, wann weitere Aufzeichnungen auftauchen, die komplizieren, zerstören oder bestätigen, was wir bereits wissen. Russland, so sagt man, hat eine unvorhersagbare Vergangenheit. Neue alte Dokumente schreiben alte neue Dokumente um. Die Entdeckung der Schriftrollen vom Toten Meer I 947 hat das Verständnis der Bibel von Grund auf revolutioniert. Wie jedes andere Objekt einer Beschreibung ist die Vergangenheit produktiv.

\section{Das Werden des Objekts}

Die Vergangenheit ist radikal unvollständig, weil die historischen Aufzeichnungen selbst historisch sind. Wissenschaftler, die an der Sozialgeschichte der Frauen interessiert sind, müssen mit dem «Mangel an Beweisen» umgehen, der eine solche Geschichte lange Zeit aus dem Blickfeld verbannt hielt. ${ }^{10}$ Historiker der Arbeit, der Kindheit, der Sklaverei, des Essens, der Mentalität, der Privatheit, des Alltagslebens und der Krankheit haben ähnliche Schwierigkeiten, Aufzeichnungen zu erschließen, die uns heute als unglaublich fruchtbar erscheinen. Vor I900 haben nur wenige Forscher davon geträumt, dass so vergängliche Dinge wie das Wetter eine schreibbare Geschichte haben könnten. Dank der Dendrochronologie (die Baumringe als Indikatoren für Jahrestemperaturen untersucht) und der Entnahme von Bohrkernen aus dem Polareis, in denen kleine Luftblasen jahrhundertelang gespeichert waren, sowie dank weiteren Technologien trägt das lebendige Feld der Klimageschichte zum wachsenden Verständnis der Geschichte des irdischen Wetters bei - etwas, das angesichts der Krise der globalen Erwärmung unmittelbare Bedeutung hat. Beweise entstehen als Antworten auf Aufmerksamkeit (Geschlechtergeschichte) oder auf technische Ausrüstung (Klimageschichte), aber vielleicht ist das letztlich die selbe Sache. Das Tagebuch von Martha Ballard wäre nur halb so aufschlussreich ohne einen Leser wie Laurel Thatcher Ulrich.
8 Lewis Mumford, Technics and Human Development, New York (Harcourt, Brace, and World) 1967 , 141-142. Ebenso: Zoe Sophia, Container Technologies, in: Hypatia 15, 2000, 181-201. Mumford würde es freuen, wenn er wüsste, dass mit DNA-Analysen bewaffnete Forscherinnen die Annahme widerlegt haben, dass jeder mit Waffen gefundene Körper ein männlicher gewesen sein soll.

9 Laurel Thatcher Ulrich, A Midwife's Tale. The Life of Martha Ballard, Based on Her Diary, 1785-1812, New York (Knopf) 1990, 24.

10 Laurel Thatcher Ulrich, WellBehaved Women Seldom Make History, New York (Knopf) 2007, XXII, 42-43, 208-220, passim. 
11 Anonym, Researchers exhume 2 Renaissance writers, in: Associated Press, 27.7. 2007. Gesehen auf www.yahoo.com am 28.7.2007.

12 Geoffrey Winthrop-Young, Memories of the Nile. Egyptian Traumas and Communication Technologies in Jan Assmann's Theory of Cultural Memory, in: New German Critique, 96, Herbst 2005, 103-133.

13 John Durham Peters, Witnessing, in: Media, Culture and Society, 23, 2001, 707-724.

14 Michael Thompson, Rubbish Theory. The Creation and Destruction of Value, Oxford (Oxford University Press) 1979.

15 Steward Brand, The Clock of the Long Now, New York (Basic Books) 1999, 103.

16 Friedrich A. Kittler, Grammophon Film Typewriter, Berlin (Brinkmann \& Bose) 1986.
So wie es a priori keine Grenze dafür gibt, was zur Botschaft werden kann - man kann nicht nicht kommunizieren -, so gibt es keine Grenze für den Reichtum der Vergangenheit.

Die Vergangenheit entfaltet sich in der Zukunft. Ein italienischer Anthropologe, der kürzlich an der Exhumierung zweier Humanisten der Renaissance teilnahm, erklärte: «Bodies are an archive of information surrounding the life and death of a person. With today's technology, we can clear up various doubts that have been passed down for centuries and we can provide answers that could not [have] been discovered years ago.» ${ }^{11}$ Pico della Mirandola ist seit I 494 tot, aber für die meiste Zeit dieser fünf Jahrhunderte war sein Körper nur ein verrottender Kadaver. Erst vor kurzem wurde er zu einem <Archiv>. Die Wissenschaftler planten eine DNA-Analyse der Körper, und natürlich ist DNA die Schlüsselfigur, dank derer aus Körpern lesbare Texte werden können. Neue Methoden der Forensik haben neue historische Speicher hervorgebracht - oder sind es alte Speicher? Das Alte entsteht ebenso wie das Neue. Der Historiker ist in der gleichen Position wie ein Zeuge: beide können nicht wissen, was die wichtigen Beweise sein werden, während die Ereignisse sich vollziehen. ${ }^{12}$ Beweise, sowohl vor Gericht als auch in der Geschichte, sind erst post facto Beweise. ${ }^{13}$ Was heute Abfall ist, mag morgen unbezahlbar sein. ${ }^{14}$

Meine Diskussion von <Geschichte〉 als einer grundsätzlichen philosophischen Abstraktion ist gefährlich, weil eine solche Konzeption selbst das Produkt eines partikulären historischen Moments ist. Und selbst diese Einsicht - dass die Vergangenheit sich in der Zukunft entfaltet - ist historisch. Nur in der Moderne mit ihrem dynamischen Anschwellen der Vergangenheit durch die Geologie, durch historisch-kritische Methoden, durch Evolutionsbiologie, Kosmologie und Archäologie konnte sich ein so intensives Bewusstsein für die Wechselwirkungen historischer Aufzeichnungen bilden. ${ }^{15}$ Dank der grafischen Revolution des I9. Jahrhunderts, in welchem Fotografie, Phonografie, Myografie, Kinematografie, Spektrografie und viele weitere Technologien mit dem Langzeitmonopol der Schrift auf kulturelle Speicherung brachen, verfügen wir über ein erweitertes Verständnis dessen, was Aufzeichnungen sein können. Dank solcher automatischen Aufzeichnungen können wir nun lesen, was nie geschrieben wurde. Analoge Medien erlauben uns, nicht-intentionale Spuren zu sichern, deren Bedeutung nicht von der symbolischen Vorbearbeitung durch Sprache abhängt. ${ }^{16}$ Das modernste an der Moderne könnte unsere Sicht auf das Vergangene sein, bis zurück zur ersten Sekunde des Big Bang. Und den besten Einblick in zeitliche Verwerfungen liefert vielleicht nicht die Medizin oder die Technologie, sondern das andauernde Durcharbeiten der Vergangenheit. Wir leben mit dem Bewusstsein, dass es vielleicht noch Medien geben wird, die aus den unscheinbarsten Bestandteilen unseres Lebens historischen Goldstaub machen werden. Die historische Aufzeichnung verschwimmt nicht nur mit der Zeit, sie kann auch an Schärfe gewinnen. 


\section{Historische Überlieferung}

Kommunikationstheorie war zunächst Claude Shannons Forschung über die Filterung und Übertragung von Signalen über eine Distanz. Zieht man die Mathematik ab, hat Geschichtsschreibung ein ähnliches Anliegen. Die Sieger mögen die Geschichte schreiben, doch es hängt vom Zufall der Natur und dem guten Willen der Nachkommenschaft ab, ob ihre Siege überleben.

Kultur und Natur formen beide die Überlieferung und Übertragung historischer Aufzeichnungen. Wie neuere Forschungen gezeigt haben, haben auch die Institutionen der kulturellen Überlieferung - Klöster, Bibliotheken, Universitäten, Museen und Archive - je eigene Interessen politischer Art. ${ }^{17}$ So viel kann von einem Auktionskatalog oder einem königlichen Archivar abhängen. Ein Zeichen der Missgunst (und womöglich die Exekution) führte in der Sowjetunion Stalins zur Auslöschung aus historischen Fotografien. Eine solche abolitio memoriae kann auch in weniger kalkulierten Weisen auftreten. Wie zugänglich werden unsere historischen Aufzeichnungen in fünfzig Jahren sein, wenn CDs bereits zu zerfallen beginnen, Terabytes von Daten auf Floppy Disks dahindümpeln und ungespielte LPs und VHS-Kassetten die Regale füllen? Weite Teile des Bestands von frühem Filmmaterial haben sich bereits aufgelöst. Sibelius hat seine achte Symphonie irgendwann in den I940er Jahren verbrannt - etwa zur gleichen Zeit, als Bachtin sein legendäres Opus Magnum geraucht haben soll, indem er aus den Manuskriptseiten Zigaretten drehte. Bevor diese Trümmer uns allzu melancholisch machen, sollten wir uns verdeutlichen, dass die Haltbarmachung von Linear B, einer Sprache, die notwendig für das Verständnis alter Schriftsysteme ist, sich einem Feuer verdankt, das zwar den Palast von Knossos zerstörte, aber die beschriebenen Lehmtafeln gebrannt hat. Das gleiche gilt für das Ugaritische. ${ }^{18}$ Die heiße Asche des Vesuv tötete das Leben der Menschen in Pompeii, aber erhielt das Leben der Stadt.

Medienwissenschaftler untersuchen die Berichterstattung in den Nachrichten, und etwas Ähnliches lässt sich in der Vergangenheit finden. Um I 800 bildete die kritische Homerphilologie und die Erforschung der Bibel das Zentrum der Revolution der Geschichtswissenschaft, und die moderne Forschung hat die Modi der Überlieferung dieser Texte nie aus dem Blick verloren. Bereits in der Antike bemerkten Koryphäen wie Cicero und Flavius Josephus die unübliche Komposition und Überlieferung Homers. Das Kennzeichen der Moderne ist die Ahnung eines unwiederbringlichen Verlustes an Quellen und Ursprüngen. Der deutsche Altphilologe Heyne schrieb Ende des r 8. Jahrhunderts: «Die Iliade, wie sie aus Homers Kopf und Munde kam, erhalten wir wohl nicht wieder; das versteht sich; eben so wenig, als die Bücher Mosis und die Propheten so, wie sie aus der Verfasser Händen kamen, wieder herzustellen sind.» ${ }^{19}$ Mit der Preisgabe der Hoffnung auf eine orale oder originäre Fülle konnten die Forscher ihr Leben dem Studium der Manuskriptvarianten und der Herausschälung des besten Textes widmen. Manchmal
17 Vgl. Wolfgang Ernst, Das Rumoren der Archive, Berlin (Merve) 2002. 18 Barry B. Powell, Writing and the Origins of Greek Literature, Cambridge (Cambridge University Press) 2002, 105 .

19 «We will not regain the lliad, as it came from Homer's mind and lips - that is clear; no more than the books of Moses and the Prophets can be restored as they came from the authors' hands." Zitiert nach Anthony Grafton, Glenn W. Most, James E. G. Zetzel, Introduction, in: Friedrich A. Wolf (Hg.), Prolegomena to Homer, Princeton (Princeton University Press) 1985,13 . 
20 Paula Fredriksen, From Jesus to Christ. The Origins of the New Testament Images of Jesus, New Haven (Yale University Press) 1988, 5 .

21 Marshall McLuhan, The Gutenberg Galaxy, Toronto (University of Toronto Press) 1962, 134.

22 Martha Howell, Walter Prevenier, From Reliable Sources: An Introduction to Historical Methods, Ithaca (Cornell University Press) 2001, 62.

23 David S. Blondheim, Les gloses françaises dans les commentaires talmudiques de Raschi, Baltimore (Johns Hopkins University Press) 1937 , Bd. 2. wurden sogar Quellentexte imaginiert, wie etwa der berühmte Text $\langle\mathrm{Q}\rangle$ (nach dem deutschen Wort Quelle), der als Bezugspunkt der Bücher der drei Apostel Markus, Matthäus und Lukas vermutet wurde. Forscher, die sich mit dem neuen Testament beschäftigen, versuchen, die Zirkulation der Predigten Jesu nachzuvollziehen: «If the report is communicated through different people over a period of time before it achieves written form (as is the case with the gospels), revision can occur at every human link in the chain of transmission. ${ }^{20}$ Das Buch, von dem man einst glaubte, es sei den Sekretären des heiligen Geistes diktiert worden, erschien nunmehr als historischer Flickenteppich: das Modern der Moderne.

Übertragung ist niemals bloß ein leerer Kanal. Medien sind ebenso wenig Röhren für Inhalt, wie Zeit ein homogenes und leeres Medium ist, das die Vergangenheit transportiert. F. A. Wolf, Begründer des Begriffs 〈Philologie〉 und einer der bedeutendsten Homer-Forscher des I8. Jahrhunderts, behauptete, dass neuere Dokumente authentischer sein könnten als alte. Er glaubte, dass er über bessere Manuskripte Homers verfüge als ein Bibliothekar in Alexandria, der Homers Schriften zwei Jahrtausende zuvor edierte. (Er wusste, dass die Vergangenheit auf ungleichzeitige Weise erscheinen kann.) Manchen Forschern erscheinen offensichtliche Übersetzungsfehler in verschiedenen Übertragungen eines Textes von großem Wert - besonders, seitdem die Vorstellung einer wortgetreuen Übersetzung selbst historisch geworden ist. Die Unterscheidung zwischen Schreiber und Autor wurde im Mittelalter selten getroffen, und vielleicht hat erst die Druckerpresse die Rede von einem «gleichmäßigen und wiederholbaren Text» in die Welt gebracht. ${ }^{21}$ Schriftliche «<errors - misspellings, grammatical faults, transpositions, even apparent omissions - can be significant historical evidence, occasionally about the politics that informed the creation of the particular text and always about the literary technology of the age. ${ }^{22}$ Die mundartlichen Randbemerkungen in hebräischen Texten des Bibelkommentators Rashi aus dem I I. Jahrhundert wurden von jüdischen Forschern lange als Kritzelei vernachlässigt, bis sie als Schatztruhe unbelegter Formen des mittelalterlichen Französisch entdeckt wurden. ${ }^{23}$ Die Analyse der Bereitstellung von Beweisen ist ein Schlüsselfaktor einer systematischen Forschung.

\section{Lückenforschung}

Vielleicht ist alle Forschung Medienforschung. Zumindest in der Evolutionsbiologie und in der Kosmologie ist dies offensichtlich - auf zwei Feldern, die tief in die Vergangenheit eindringen und Übertragungsprozesse in Interpretationshilfen verwandeln. Das Kapitel On the Imperfection of the Geological Record in Darwins Origin of Species (I 859) stellt die ungenügenden Beweise einer vorübergehenden Verbindung zwischen Arten vor. Diese missing links sind aber, so Darwin, nicht durch Fossilien nachzuweisen - nicht weil sie nie entstanden sind, sondern weil die Aufzeichnung beschädigt oder unvollständig ist. Die Aufzeichnung der 
Geschichte des Lebens ist Gegenstand der verschüttenden Prozesse der Erdgeschichte, von der Erosion bis zum Vulkanausbruch. Wie Garfinkel liest Darwin diese Aufzeichnungen unter Verweis auf die Prozesse, die sie geformt haben. Um die Einheitlichkeit seiner Annahmen angesichts des katastrophalen Zustands der Materialien aufrechtzuerhalten, argumentierte Darwin mit Übertragungslücken aus der Vergangenheit und nicht mit der Vergangenheit selbst. Wäre die Übertragung vollständiger, so behauptet er, würden wir bessere Hinweise auf die von ihm postulierten Übergangsformen haben. Die Suche nach Beweisen für die Verbindung zwischen den Arten in den geologischen Materialien wäre für Darwin in etwa so, als würde man die antiken Olympischen Spiele auf dem Video mit den größten Momenten der Sportgeschichte suchen.

In der Kosmologie liefern potentielle Störungen der Übertragungen entscheidende Daten für die Geschichte des Universums. Wenn wir tief in den Weltraum blicken, schauen wir in der Zeit zurück. Das Licht, das unsere Retina trifft, begann die Reise von seiner Quelle möglicherweise vor Äonen. Da wir in einem sich ausdehnenden Universum leben, ist das Licht, das am weitesten reist, den heftigsten Störungen ausgesetzt. Gemäß dem Doppler-Effekt - Wellen, die sich von einem stillstehenden Beobachter wegbewegen, werden in größere Wellenlängen gedehnt (mit entsprechend niedrigeren Frequenzen) - ist Licht aus weit entfernten Quellen in den roten, niederfrequenten Bereich verschoben. Die Rotverschiebung erlaubt es, die Geschwindigkeit solcher Quellen und damit indirekt ihr Alter zu messen. Je früher eine Lichtübertragung begann, desto schneller entfernt sich die Quelle, weil die größten Ausdehnungsgeschwindigkeiten vermutlich am Anfang des Big Bang herrschten. Je weiter wir in den Weltraum blicken können, desto weiter blicken wir in der Zeit zurück, und je roter das Licht, desto weiter blicken wir im Raum. Das Universum ist von Innen nach Außen gekehrt: seine entferntesten Winkel bilden seine jüngsten Teile - aber für uns sind sie die ältesten. Anstatt Rotverschiebungen als verzerrte Übertragungen zu verstehen, realisierten Astronomen wie Edwin Hubble, dass solche Verzerrungen als Beweise für die Geschichte des Universums gelesen werden können. Er lernte, den bias der Übertragung als Index für die Vergangenheit zu lesen. Heute werden Rotverschiebungen routinemäßig genutzt, um das Alter von astronomischen Daten zu messen. Das ist die gleiche interpretative Strategie wie das Graben in den Kommentaren Rashis. ${ }^{24}$

Im I9. Jahrhundert teilen hermeneutische Geschichtskonzepte und das Kommunikationsverständnis des Spiritismus' die Träume von einer vollständigen oder perfekten Übertragung. Übertrieben schematisch gefasst, entsprach der Historismus als leitende Idee der Geschichtsschreibung seit dem i 9. Jahrhundert dem Ideal einer Zeitreise, dem Errichten einer so perfekten Rekonstruktion der Vergangenheit, dass der Historiker völlig in dieser vergangenen Welt aufginge. Im Spiritismus, der zur selben Zeit die populären Konzepte der Kommunikation prägte, führt dieser Traum zu Telepathie, zur mentalen Kommunion, die den Ab-
24 Dieses Argument entwickle ich in: Space, Time, and Communication Theory, in: Canadian Journal of Communication, 28, 2003, 397-411. 
25 Vgl. John Durham Peters, Speaking into the Air, Chicago (University of Chicago Press) 1999. 26 Josiah Royce, The Religious Aspect of Philosophy, Gloucester/MA (Peter Smith) $1885 / 1965$.

27 Walter Benjamin, Über den Begriff der Geschichte, in: ders., Gesammelte Schriften, Frankfurt/M. (Suhrkamp) 1980, Bd. 2, 691-704.

28 Michael Pollan, The Omnivore's Dilemma. A Natural History of Four Meals, New York (Penguin) 2006. grund zwischen zwei Geistern verschwinden lassen würde. Beide Träume weben ein Begehren nach einem perfekten Medium der Kommunikation fort, das die Abgründe transzendieren würde. Beide Träume entstehen als Reaktion auf die verdichtenden Apparate der Geschichte und der Kommunikation. Die kritische Methode, die um I80o in Deutschland aufkam, lehrte, dass Dokumente, Archive und Quellen gerade nicht transparent sind und keine neutralen Kanäle der Kommunikation darstellen. Die moderne Geschichtsforschung ist bis zu einem gewissen Grad immer eine Reflektion auf die Bedingungen ihrer eigenen (Un-) Möglichkeit. Quellenkritik ist das Gütesiegel des Historismus und Historiker wären die ersten, die zugeben würden, dass die Vergangenheit letztlich unerreichbar ist. Moderne Frauen und Männer mussten sich um I900 mit Quellenkritik beschäftigen, während sie sich an die Kommunikation mit Telegramm, Telefon und Schnellpost gewöhnten. Sie mussten lernen, Medieneffekte (etwa die Verzögerung in der Zustellung) und Auswahlmöglichkeiten (etwa der Auswahl, nicht $\mathrm{zu}$ antworten) zu trennen. Als elektrische und andere Medien das Raum-ZeitKontinuum aufrissen, versprachen beide, die bekannten Abstände zu überwinden und drohten, die gewohnte Intimität zu zerstören. Unsere Vorstellungen von Geschichte und Kommunikation reflektieren zugleich die moderne Hoffnung auf Überschreitung und ihre Furcht vor Zusammenbruch. ${ }^{25}$

Es ist allzu einfach, diese Träume zu kritisieren, aber Übertragung ist in gewisser Hinsicht eine zu wichtige Kategorie für Historiker und Medientheoretiker, um sie beiseite zu lassen. «The possibility of error» ist ein Anker in der vernünftigen Ordnung des Universums, wie der große, vergessene Josiah Royce behauptete. ${ }^{26}$ Das Interesse an korrekter Übertragung wird eher vom ethischen Bemühen um Respekt vor dem Anderen bewegt als von einer epistemischen Suche nach einer Reinheit des Zugriffs oder einer Fülle der Präsenz. Den Holocaust zu leugnen ist nicht einfach nur dumm - es ist unmoralisch. Es ist weniger ein Denkfehler als eine Störung des Rechtsempfindens. Wenn wir die Vergangenheit studieren, haben wir es mit der grundlegendsten, aber auch fragilsten aller kommunikativen Beziehungen zu tun: der zwischen den Lebenden und den Toten. ${ }^{27}$ Die Aufgabe des Historikers ist nicht, die Toten zu töten. Seine Aufgabe ist, zu erkennen, wie sie immer und immer wieder in der Erinnerung geboren werden, und zu erkennen, wie die Welt immer wieder mit neuen alten Dingen angefüllt wird. Das ist die zugrunde liegende Ethik, die das Studium der Geschichte und der Kommunikation und den Medien vereint.

\section{Historische Interpretation}

Die Gefahr eines Schlaganfalls durch eine Informationsüberdosis gab es in vielen historischen Momenten - daran ist nichts neu. Aber wir leben in einer Zeit höchster archivarischer Sensibilität, die zum Teil auf das Internet zurückzuführen ist. Historiker müssen sich damit auseinandersetzen, was Michael Pollan in einem anderen Kontext «the omnivore's dilemma ${ }^{28}$ genannt hat. Jede Nachforschung 
ist potentiell bodenlos. Die fraktale Geometrie zeigt, dass jede Vergrößerungsstufe eine kontingente Wahl ist. Die Länge der Küste Großbritanniens ist eine Funktion des Messinstruments. ${ }^{29}$ Jeder Dissertant verzweifelt daran, dass jeder Satz seiner Dissertation eine eigene Dissertation sein könnte, und dass seine ganze Dissertation ein Satz in der Dissertation eines anderen sein wird. Was gespeichert wird, ist ein winziger Teil dessen, was geschehen ist, und was uns überliefert wird, ist wiederum nur ein Bruchteil dessen - aber die Entscheidungen, die wir mit jeder Interpretation treffen, implizieren eine weitere Selektion. (Natürlich ist Wahl nicht nur Verkleinerung; Speicherung, Übertragung und Interpretation setzen unter Umständen erst zusammen, «was passiert ist».) Wir haben keine andere Wahl als auszuwählen, weil unsere Aufmerksamkeit begrenzt und unser Leben endlich ist. Wir können große Geschichten kleiner Dinge schreiben - wie des Kabeljaus, des Salzes oder der Unterschrift - oder kleine Geschichten großer Dinge - wie der Zeit oder der ersten Sekunde des Universums -, aber kein Autor kann in einer Lebensspanne eine Universalgeschichte verfassen. Die Auswahl ist nicht nur eine Frage des Herangehens an einen Materialkorpus, sondern betrifft die existenzielle Entscheidung dafür, womit man seine Zeit verbringt. Interpretation ist, zumindest zum Teil, ein Budgetierungsverfahren für Zeit, Raum und Energie. Die Geschichtsschreibung selbst ist Teil der Geschichte des Menschen. Unser eigenes Eingebettetsein in Geschichte formt unsere Weisen des Schreibens viel grundsätzlicher, als wir meist vermuten.

In der Kosmologie unterstreicht das «anthropische Prinzip» genau diese Verbindung zwischen Beobachterposition und Einsicht. Das Prinzip besagt, dass nur eine bestimmte Art von Universum uns als Beobachter ermöglichen konnte. Das Universum musste alt und kühl genug sein, um die komplexen Chemikalien zu erzeugen, die die notwendigen Zutaten für intelligentes Leben, wie wir es kennen, darstellen. Die Möglichkeit, dass wir überhaupt etwas über das Universum wissen, erfordert eine Art von Universum, in der wir existieren können. In dem Moment, in dem das Universum genügend differenziert ist, um Lebensformen zu beherbergen, die es bis zu einem gewissen Grad verstehen können, muss es notwendigerweise ein kalter, leerer, dunkler Ort geworden sein (wenn man eine organische Basis für intelligentes Leben annimmt). «What we can expect to observe must be restricted by the condition necessary for our presence as observers.» ${ }^{30}$ Das anthropische Prinzip weist auf ein Zusammenfallen unserer existenziellen Situation mit unseren erkenntnistheoretischen Möglichkeiten hin. Als Historiker des Universums sind wir Elemente in dessen Geschichte. Nur an einem bestimmten Punkt der Geschichte des Universums können wir überhaupt zu seinen Historikern werden. Dass wir Übertragungen aus der Tiefenzeit und dem Tiefenraum des Universums empfangen können, ist unserer Position in Zeit und Raum geschuldet. Unsere Fähigkeit, solche Botschaften lesen zu können, wird von den historischen Prozessen geformt, die diese Nachrichten erzeugt haben. Historiker haben den Zugang zur Geschichte, den die Geschichte ihnen zu haben gewährt.
29 Benoit Mandelbrot, How Long is the Coast of Britain. Statistical SelfSimilarity and Fractional Dimension, in: Science $156,1967,636-638$. 30 John D. Barrow, The Constants of Nature, New York (Pantheon) 2002, 160-176; Zitat von Brandon Carter aufS. 162 . 
Innerhalb dieser Grenzen sind sie aber gezwungen, schwierige Entscheidungen zu treffen.

Ereignisse können von einer inhärenten Unbestimmtheit sein. Die Ungewissheit der Vergangenheit resultiert nicht nur aus der Beschränktheit unserer Perspektive, sondern aus der Unbestimmtheit dessen, was geschieht. Wir können zwar fantasieren, dass eine immer perfektere Maschinerie der Aufzeichnung selbst die Bewegung jedes Moleküls speichern könnte - aber das würde nur die Sprungkraft im Inneren der Dinge zeigen. Dass die Dinge umso unschärfer erscheinen, je besser sie dokumentiert sind, können wir auch von der Quantenphysik lernen. Die Wirklichkeit kann so vage sein wie Texte, und Texte können so unentwirrbar sein wie die Wirklichkeit. Beschreibungen können nicht nur so unerschöpflich sein, weil die Sprache generativ ist, sondern weil das Universum unvollständig ist. Ebenso wie wir oft nicht wissen, was wir meinen, wenn wir sprechen, ist sich vielleicht auch das Universum seiner selbst nicht sicher.

\section{Postscriptum}

Insofern die Geschichtswissenschaft, reflexiv betrachtet, Mediengeschichte ist, haben wir Einiges an Arbeit vor uns. Eine Forschungsachse ist die Zeit selbst. Innis wurde bislang eher zitiert, als in seinem Bemühen nachgeahmt, alte und moderne Kulturen zu vergleichen. Die Prähistorie liefert ein reiches Feld, das lange Zeit in der alleinigen Hand der Anthropologen war: die Domestizierung des Feuers, die Institutionen der Verwandtschaft, Körpertechniken und Stimmpraktiken, die Kunst des Sprechens und Schreibens. Die Domestizierung von Pflanzen und Tieren, das Kochen und die Erziehung von Kindern, die Techniken der Navigation und der Zeitmessung, Rituale und die Kunst der Erinnerung sind essentielle Bestandteile der langen Geschichte der Kommunikation. Das gesammelte Archiv der Menschheit - religiös, philosophisch, juristisch, literarisch und künstlerisch - birgt einen reichen Schatz an Medienpraktiken. Auch Mediengeschichten außerhalb Europas und Nordamerikas warten auf ihre Historiker. Die Globalisierung der wissenschaftlichen Kommunikation in der Gegenwart könnte uns helfen, eine globalere Vergangenheit zu erschaffen. Der Druck, interdisziplinär zu arbeiten, sollte uns über den Abgrund zwischen Natur- und Geisteswissenschaften hinweghelfen zu einer Wissenschafts- und Technikgeschichte. Von

31 Ich entwickle diesen Gedanken in: Strange Sympathies. Horizons of German and American Media Theory, in: Frank Kelleter, Daniel Stein (Hg.), American Studies as Media Studies, Heidelberg (Winter) 2008.

32 Ich danke Georgina Born, Samuel McCormick, Benjamin Peters, Peter Simonson und Geoffrey Winthrop-Young für hilfreiche Kommentare und Korrekturen.
Peirce stammt das Diktum, dass die Metaphysik der Affe der Mathematik ist, und Physik und Mathematik stehen hinter vielen Kommunikationsweisen und Medientechniken. Letztlich sollten wir, um Innis zu folgen, unseren Medienbegriff erweitern. ${ }^{31} \mathrm{Um}$ nur Einiges zu nennen: Astrolabium, Batterie, Chor, Ding, Eid, Feuer, Glocke, Horn, Insel, Jagd, Karte, Leuchtturm, Metronom, Namen, Observatorium, Punkt, Quantum, Röntgenstrahlung, Schiff, Tastatur, Unterschrift, Vokal, Wachs, X-Achse, Yoktosekunde, Zeuge. Die Zukunft der Geschichte der Medien wartet auf uns. ${ }^{32}$ 